Die Zukunft des Sozial- und Steuerstaates

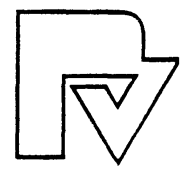




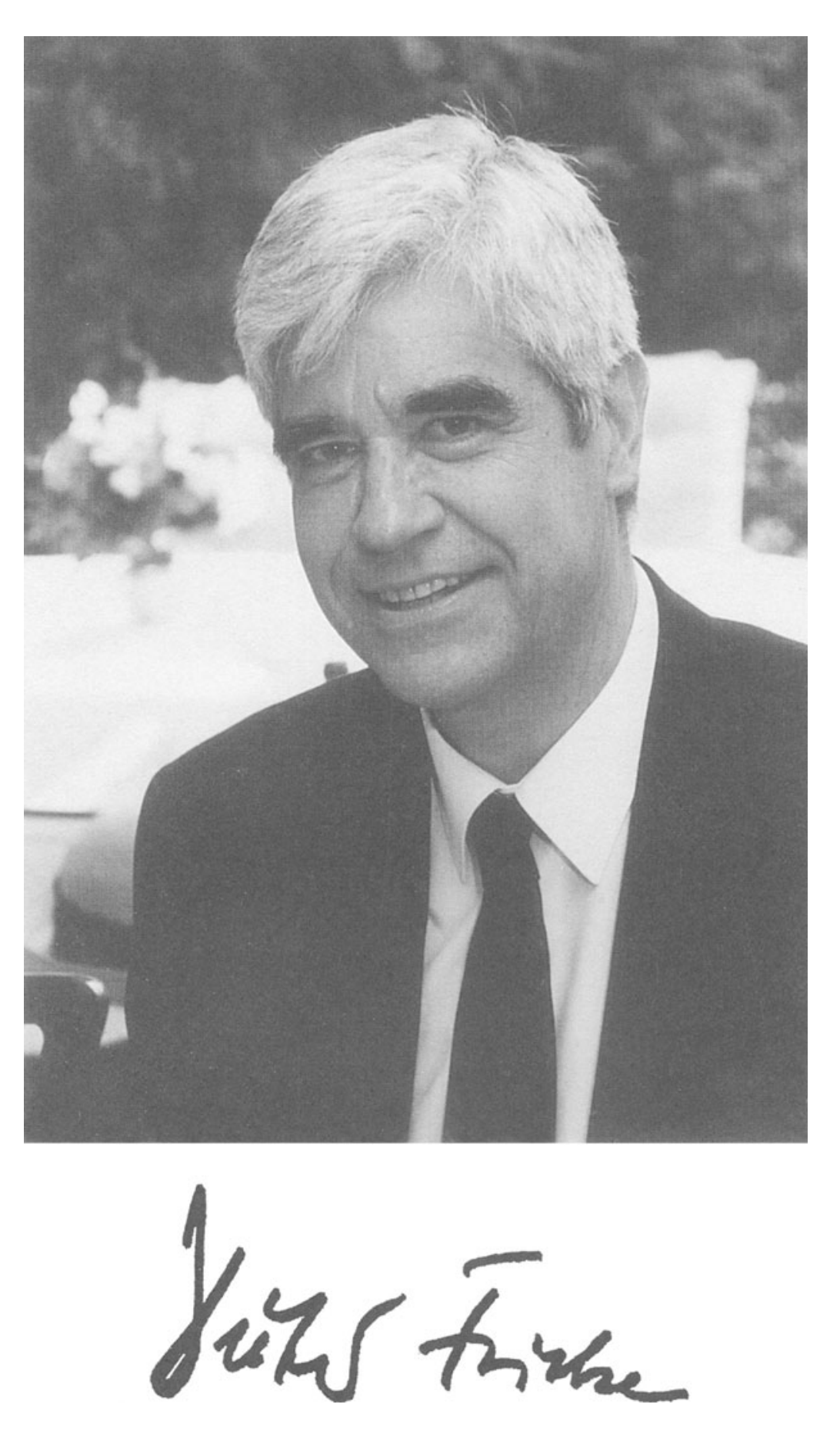


Fritz Söllner · Arno Wilfert (Hrsg.)

\section{Die Zukunft des Sozial- und Steuerstaates}

Festschrift zum 65. Geburtstag von Dieter Fricke

Mit 35 Abbildungen und 38 Tabellen

Springer-Verlag Berlin Heidelberg GmbH 


\section{Professor Dr. Fritz Söllner \\ Technische Universität Ilmenau \\ Fachgebiet Finanzwissenschaft \\ 98684 Ilmenau \\ Deutschland}

Dr. Arno Wilfert

C-Quential GmbH

Martin-Luther-Platz 26

40212 Düsseldorf

Deutschland

ISBN 978-3-642-63308-9

DOI 10.1007/978-3-642-57606-5

ISBN 978-3-642-57606-5 (eBook)

Die Deutsche Bibliothek - CIP-Einheitsaufnahme

Die Zukunft des Sozial- und Steuerstaates: Festschrift zum 65. Geburtstag von Dieter Fricke / Hrsg.: Fritz Söllner; Arno Wilfert. - Springer-Verlag Berlin Heidelberg GmbH

Dieses Werk ist urheberrechtlich geschützt. Die dadurch begründeten Rechte, insbesondere die der Übersetzung, des Nachdrucks, des Vortrags, der Entnahme von Abbildungen und Tabellen, der Funksendung, der Mikroverfilmung oder der Vervielfältigung auf anderen Wegen und der Speicherung in Datenverarbeitungsanlagen, bleiben, auch bei nur auszugsweiser Verwertung, vorbehalten. Eine Vervielfältigung dieses Werkes oder von Teilen dieses Werkes ist auch im Einzelfall nur in den Grenzen der gesetzlichen Bestimmungen des Urheberrechtsgesetzes der Bundesrepublik Deutschland vom 9. September 1965 in der jeweils geltenden Fassung zulässig. Sie ist grundsätzlich vergütungspflichtig. Zuwiderhandlungen unterliegen den Strafbestimmungen des Urheberrechtsgesetzes.

(C) Springer-Verlag Berlin Heidelberg 2001

Originally published by Physica-Verlag Heidelberg 2001

Softcover reprint of the hardcover 2 nd edition 2001

Die Wiedergabe von Gebrauchsnamen, Handelsnamen, Warenbezeichnungen usw. in diesem Werk berechtigt auch ohne besondere Kennzeichnung nicht zu der Annahme, dass solche Namen im Sinne der Warenzeichen- und Markenschutz-Gesetzgebung als frei zu betrachten wären und daher von jedermann benutzt werden dürften.

Umschlaggestaltung: Erich Kirchner, Heidelberg

SPIN $10796158 \quad 88 / 2202-543210$ - Gedruckt auf säurefreiem Papier 


\section{Dieter Fricke zum 65. Geburtstag}

Dieter Fricke wurde am 2. April 1936 in Dortmund geboren. Sein Studium der Betriebswirtschaftslehre und Rechtswissenschaft absolvierte er in Heidelberg, Freiburg und Köln. Von 1962 bis 1973 forschte und lehrte er an der Universität zu Köln, wo er als wissenschaftlicher Assistent am Finanzwissenschaftlichen Seminar von Günter Schmölders arbeitete. 1966 promovierte er dort zum Dr. rer. pol. Das Thema seiner Dissertation lautete „Das Sparverhalten der privaten Haushalte in der Bundesrepublik Deutschland“. Im Rahmen eines Habilitationsstipendiums der Deutschen Forschungsgemeinschaft habilitierte er sich 1976 an der Universität zu Köln mit dem Thema „Verteilungswirkungen der Inflation“; sein Habilitationsvortrag hatte „Steuerstruktureffekte in einer wachsenden Wirtschaft" zum Gegenstand.

Dieter Frickes wissenschaftlicher Ansatz wurde stark von seinem Lehrer Günter Schmölders geprägt, der als Mitbegründer der institutionell orientierten Kölner Schule der Finanzwissenschaft neben der reinen Finanztheorie immer auch nichtökonomische - vor allem politische, historische, gesellschaftliche und psychologische - Faktoren berücksichtigte. Auch Dieter Fricke hat immer eine multidisziplinäre Finanzwissenschaft betrieben, was nicht nur in seiner Habilitationsschrift, sondern auch in seinen späteren Veröffentlichungen zum Ausdruck kommt.

Nach Lehrstuhlvertretungen an den Universitäten Trier und Siegen nahm er 1980 einen Ruf auf den Lehrstuhl für Finanzwissenschaft an die Universität Bayreuth an, deren Rechts- und Wirtschaftswissenschaftliche Fakultät sich zu jener Zeit noch im Aufbau befand. Hier engagierte sich Dieter Fricke beim Auf- und Ausbau der Fakultät und trug maßgeblich zum guten Ruf dieser noch jungen Universität bei. Nach dem Fall der Mauer leistete er erneut Pionierarbeit und übernahm ohne langes Zögern zusätzlich zu seinen Verpflichtungen in Bayreuth eine Gastprofessur an der Technischen Universität Chemnitz-Zwickau. Er setzte sich generell mit großem Enthusiasmus für den Aufbau der wirtschaftswissenschaftlichen Fakultäten an den Universitäten in den neuen Bundesländern ein.

Dieter Fricke ist Mitglied im Verein für Socialpolitik, im Leitungsgremium des Bayreuther Instituts für Afrika-Studien, der Forschungsstelle für Raumanalysen, Regionalpolitik und Verwaltungspraxis an der Universität 
Bayreuth sowie im Bayreuther Graduiertenkolleg „Interkulturelle Beziehungen in Afrika“. Seit 1994 ist er Sprecher des Bayerischen Wirtschaftsund Sozialwissenschaftlichen Fakultätentages und seit 1997 Vorstandsmitglied des Bayerischen Forschungsverbundes Forarea. Von 1992 bis 1994 war er Dekan und von 1994 bis 1996 Prodekan der Rechts- und Wirtschaftswissenschaftlichen Fakultät der Universität Bayreuth.

Seit 1984 engagiert sich Dieter Fricke in der Afrikaforschung. So war er von 1984 bis 1997 Mitglied und Projektleiter im Sonderforschungsbereich 214 der Deutschen Forschungsgemeinschaft „Identität in Afrika - Prozesse ihrer Entstehung und Veränderung“. Die Schwerpunkte seiner Forschungsarbeiten lagen zunächst im Bereich des einheimischen afrikanischen Unternehmertums. Ab 1995 - im Rahmen seiner Arbeit für den Bayerischen Forschungsverbund Forarea - spielte der Zusammenhang zwischen infrastrukturellen Defiziten und mangelnder wirtschaftlicher Entwicklung eine zunehmend wichtigere Rolle. Dieter Fricke führte mit seinem interdisziplinären Team mehrere Primärerhebungen und Fallstudien in Nigeria, Tanzania und später in Südafrika durch.

Dieter Fricke hat stets ein offenes Ohr und Verständnis für die Belange der Studenten, gleich ob diese im Zusammenhang mit dem Studium stehen oder eher persönlicher Art sind. Auch in den heutigen Zeiten der Massenuniversität ist er immer bemüht, ein gutes Verhältnis zu seinen Studenten aufzubauen und zu erhalten. Er bewahrt bei aller Arbeit seinen Sinn für Humor, der in seiner rheinländischen Herkunft begründet zu sein scheint: Vorlesungen am Rosenmontag führt er zur Freude seiner Studenten regelmäßig im Karnevalskostüm durch.

Dieter Fricke ist ein Mensch, der immer nach vorne blickt, bereit ist anzupacken, wenn es darum geht Aufbauarbeit zu leisten oder komplexe Forschungsvorhaben in schwierigem Umfeld durchzuführen. Er bemüht sich immer um eine unvoreingenommene Betrachtung und wehrt sich vehement gegen einseitige Urteile und voreilige Schlußfolgerungen. Er versteht es außerdem überaus gut, aktuelle Problemstellungen mit historischen Fakten zu verknüpfen und vor einem interdisziplinären Hintergrund zu analysieren; nicht zuletzt deshalb ist er ein anregender und kenntnisreicher Diskussionspartner.

Die offene und positive Grundeinstellung Dieter Frickes verspürten auch seine Kollegen, Studenten und Doktoranden sowie die Mitarbeiter seines Lehrstuhls. Es überrascht deshalb nicht, daß unser Vorhaben, eine Festschrift zu Ehren von Dieter Fricke herauszugeben, auf große Resonanz stieß und sich eine ganze Reihe renommierter Autoren gerne zur Mitarbeit bereit fand. 
Die vorliegenden 19 Beiträge betreffen die Finanzwissenschaft, die Sozialpolitik und die Geldpolitik. Sie decken damit die Lehrgebiete Dieter Frickes sehr schön ab - was für seine Forschungsarbeiten insofern nicht gilt, als daß wir leider eine seiner Hauptinteressen - die Afrikaforschung - im Rahmen unserer Festschrift aus konzeptionellen Gründen nicht berücksichtigen konnten. Hierfür möchten wir den Jubilar an dieser Stelle um Verständnis bitten.

Im folgenden soll ein kurzer Überblick über den Inhalt der Festschrift gegeben werden, die in die drei Abschnitte „Neue Herausforderungen für die Finanzpolitik“, „Der Sozialstaat in der Krise“ und „Der Euro und die europäische Geldpolitik“" aufgeteilt ist.

Den finanzwissenschaftlichen Teil eröffnet Guy Kirsch mit seinem Kapitel „Der Dritte Sektor - eine Chance für den Staat?“, in dem er die Rolle des Dritten Sektors, d.h. der nichtstaatlichen und nichtmarktlichen Öffentlichkeit diskutiert. Er kommt zu dem Ergebnis, daß Einrichtungen des Dritten Sektors vor allem auf lokaler und supranationaler Ebene als Chance begriffen und dementsprechend an Bedeutung gewinnen werden - was letztlich zu Lasten des Nationalstaats gehen wird.

Eines der Hauptprobleme, mit denen sich der Nationalstaat konfontiert sieht, ist das seiner Finanzierung. So wird behauptet, daß der Globalisierungsdruck bzw. der internationale Standortwettbewerb eine Senkung von Steuer- und Abgabenquote und eine Verschiebung weg von direkten, hin zu indirekten Steuern erzwingen würde. Da Deutschland sich diesem Wettbewerb bisher nicht gestellt hätte, seien nun schnelle und radikale Reformschritte in bezug auf Steuerhöhe und -struktur notwendig. Diese und andere Thesen überprüfen Dieter Ewringmann und Klaus Mackscheidt in Kapitel 2 („Die Entwicklung der Steuerstrukturen in internationalen Vergleich“). Ihre empirische Analyse zeigt, daß die behauptete Entwicklung mitnichten stattgefunden hat: Bei den wichtigsten Industriestaaten lassen sich weder signifikante Rückgänge der Steuer- bzw. Abgabenquote noch ein Anwachsen der Bedeutung indirekter Steuern feststellen; deshalb fällt Deutschland, was Steuerhöhe und -struktur angeht, im internationalen Vergleich keineswegs aus dem Rahmen.

Auch Jochen Sigloch befaßt sich mit der Besteuerung in Deutschland allerdings aus steuersystematischer Sicht. Im dritten Kapitel („Die Unternehmensbesteuerung - Reformstand und Reformbedarf") analysiert er die Unternehmensbesteuerung nach Abschluß der jüngsten Reformrunde. Er sieht hier weiterhin erheblichen Handlungsbedarf und fordert, neben einer Neufassung der Unternehmensabgrenzung und dem Abbau diskriminieren- 
der Sondersteuern, die Revision des von der Wissenschaft heftig kritisierten Systemwechsels zum Halbeinkünfteverfahren.

Nicht mit der Einnahmen-, sondern mit der Ausgabenseite öffentlicher Haushalte beschäftigen sich Dietrich Dickertmann und Annemarie Leiendecker in Kapitel 4 (,Der Beihilfenbericht der Europäischen Union: Darstellung und Bewertung"). Sie widmen sich dem Beihilfenbericht der Europäischen Union - ein Gegenstand, der bislang von Seiten der Wissenschaft nur wenig Aufmerksamkeit erfahren hat, diese aber durchaus verdient: Denn die Berichterstattung der Europäischen Kommission über die Vergabe (nationaler und gemeinschaftlicher) Subventionen an Unternehmen (welche im europäischen Sprachgebrauch Beihilfen genannt werden) weist schwerwiegende Mängel formaler und inhaltlicher Art auf. Um Abhilfe zu schaffen und eine sinnvolle Interpretation des Beihilfenberichts zu ermöglichen, machen die Autoren eine Reihe beachtenswerter Verbesserungsvorschläge.

Was die (nationalen) Subventionen in Deutschland angeht, so war und ist die Eisenbahn einer der wichtigsten Empfänger derselben. An diesem Beispiel demonstrieren Helmut Gröner und Andreas Knorr in Kapitel 5 („Öffentliche Unternehmen in der Marktwirtschaft: Das Beispiel Eisenbahn") die ordnungstheoretische Problematik öffentlicher Unternehmen, die als teuerstes und ineffizientestes Mittel zur Verwirklichung gemeinwirtschaftlicher Ziele gelten können. So weise die Bahn in Deutschland auch nach ihrer (nur formalen) Privatisierung eine sehr geringe Wettbewerbsfähigkeit auf wofür die in wichtigen Punkten inkonsequente Bahnstrukturreform von 1994 zu einem Großteil verantwortlich sei.

Wie die Misere der Deutschen Bahn ist auch die Reform der Finanzverfassung ein finanz- bzw. wirtschaftspolitischer „Dauerbrenner“. Die geringe Effizienz des gegenwärtigen Systems kritisiert Horst Zimmermann in Kapitel 6 („Ein Hebesatzrecht für Länder und Gemeinden?“), wobei er sich auf die Theorie des Fiskalföderalismus und die Theorie des Wettbewerbsföderalismus stützt. Vor allem auf der Einnahmenseite scheint eine stärker wettbewerbliche Ausrichtung der Finanzverfassung dringend vonnöten. $\mathrm{Zu}$ diesem Zweck empfiehlt der Autor als ersten, am ehesten politisch realisierbaren Schritt die Ergänzung des bestehenden Verbundsteuersystems durch Hebesatzrechte für Länder und Gemeinden.

Ebenfalls der Finanzverfassung ist Kapitel 7 (,Ein Weg aus der Krise des deutschen Länderfinanzausgleichs") von Fritz Söllner gewidmet: Mit dem Länderfinanzausgleich steht der wohl am heftigsten umstrittene Bestandteil der Finanzverfassung zur Diskussion. Nach einer kritischen Auseinandersetzung mit dem gegenwärtigen Länderfinanzausgleich entwickelt der Verfasser einen Reformvorschlag, der sowohl den Vorgaben des Bundesverfas- 
sungsgerichts als auch den finanzwissenschaftlichen Anforderungen an ein Finanzausgleichssystem Rechnung trägt.

Mit Kapitel 8 („Der Einfluß des Sozialstaatsprinzips auf die Entwicklung des deutschen Länderfinanzausgleichs") beschließt Peter Thuy den finanzwissenschaftlichen Teil und schlägt gleichzeitig eine Brücke zu den sozialpolitischen Fragestellungen des folgenden Abschnitts. Der Autor sieht die Ursache für die Probleme des gegenwärtigen Systems des Länderfinanzausgleichs in der immer extensiveren Auslegung des Sozialstaatsprinzips: So wie dies in der Sozialpolitik zu einer Erfassung immer weiterer Bevölkerungskreise und einer Ausdehnung des Leistungsumfangs geführt habe, so habe dies auch einen Wandel im Charakter des Länderfinanzausgleichs bewirkt - an die Stelle der Hilfe in Notsituationen und der Herstellung von Chancengleichheit sei eine immer weitergehende Nivellierung der Finanzkraft getreten, die mit einer starken Ausweitung des Ausgleichsvolumens einhergegangen sei.

Dem Sozialstaatsprinzip widmen sich auch Peter Oberender und Stephan Ruckdäschel in Kapitel 9 („Sozialstaatsprinzip und Wirtschaftsordnung"), das am Anfang des sozialpolitischen Abschnitts steht. Sozialpolitische Eingriffe des Staates könnten zwar - nicht zuletzt wegen ihrer systemstabilisierenden Wirkung - auch in marktwirtschaftlichen Ordnungen gerechtfertigt werden, doch bestünde immer die Gefahr der Zerstörung solcher Ordnungen durch zu weitgehende Eingriffe des Sozialstaates. Dem könne nur begegnet werden durch eine ordnungskonforme, d.h. die individuelle Handlungs- und Entscheidungsfreiheit so weit als möglich erhaltende Sozialpolitik.

Die folgenden vier Beiträge befassen sich mit den großen Problemfeldern der gegenwärtigen Sozialpolitik: der Gesundheits-, der Renten- und der Beschäftigungspolitik. Zunächst diskutiert Frank Daumann in Kapitel 10 (,Zur Notwendigkeit der Harmonisierung der staatlichen Krankenversicherungssysteme in Europa") die Frage, ob die staatlichen Krankenversicherungssysteme innerhalb der EU harmonisiert werden sollten. Er gelangt zu dem Ergebnis, daß eine Harmonisierung nur dann notwendig wäre, wenn es für alle EU-Bürger die Möglichkeit eines unbeschränkten Wechsels zwischen den verschiedenen nationalen Krankenversicherungssystemen gäbe, da diese dadurch in ihrer Existenz gefährdet werden würden. Solange dies nicht der Fall sei, könne und solle der Systemwettbewerb beibehalten werden - vor allem, weil dieser eine stärkere Orientierung an den individuellen Präferenzen bewirke als ein europaweit einheitliches Krankenversicherungssystem.

In Kapitel 11 (,Die Rentenreform in Deutschland - eine kritische Würdigung") legt Bert Rürup die Gründe für die Notwendigkeit einer umfassenden Rentenreform dar und setzt sich mit dem Rentenreformkonzept der 
Bundesregierung auseinander, welches im wesentlichen in der Ergänzung des Umlangeverfahrens durch eine zusätzliche Privatvorsorge nach dem Kapitaldeckungsverfahren besteht. Dieses Konzept wird vom Autor überwiegend positiv beurteilt, wenngleich er die Freiwilligkeit der Zusatzsicherung bemängelt.

Die Frage, ob die deutsche Beschäftigungspolitik von ausländischen Erfahrungen profitieren kann, stellt sich Klaus Schuberth in Kapitel 12 (,Wege zu mehr Beschäftigung in Deutschland - ausländische Erfahrungen als Vorbild?"). Vorbildcharakter hätten vor allem die USA, Großbritannien, Irland, Dänemark und die Niederlande, deren Arbeitslosenquoten z.T. deutlich unter der deutschen lägen. All diesen Ländern gemein seien lohnpolitische Zurückhaltung, flexible Arbeitsmärkte und - zumindest zeitweise eine nachfragestützende Haushaltspolitik. Erfolge bei der Bekämpfung der Arbeitslosigkeit würden sich also nicht durch Einzelmaßnahmen, sondern nur durch ein abgestimmtes Vorgehen in allen relevanten Politikbereichen erzielen lassen - eine Strategie, die es auch in Deutschland umzusetzen gelte.

Umstritten ist in diesem Zusammenhang vor allem, welche Rolle der Staat bei der Lohnfindung spielen sollte. Gegenwärtig erhofft sich die Öffentlichkeit einiges vom Einwirken des Staates auf die Tarifvertragsparteien im Rahmen des „Bündnisses für Arbeit“. Thomas Pfahler gibt in Kapitel 13 (,Die staatliche Zwangsschlichtung als Ausweg aus Tarifkonflikten?") zu bedenken, daß staatliche Eingriffe in die Tarifautonomie zu unerwünschten Ergebnissen - wie abnehmende Einigungsbereitschaft der Tarifvertragsparteien und zunehmende Politisierung der Tarifverhandlungen - führen können, was er am Beispiel der staatlichen Zwangsschlichtung in der Weimarer Republik erläutert.

In dem den sozialpolitischen Teil abschließenden Kapitel 14 („Karitative Organisationen und die Krise des Sozialstaats") greifen Klaus Tiepelmann und Gregor van der Beek die im ersten Kapitel grundsätzlich diskutierte Thematik des „Dritten Sektors“ wieder auf. Sie fragen, ob und inwieweit eine der wichtigsten zum Dritten Sektor gehörenden Gruppen von Institutionen - die karitativen Organisationen - dazu beitragen kann, die gegenwärtige Krise des Sozialstaats zu lösen. Mit Hilfe der Theorie der Parafiski zeigen die Verfasser, daß bestimmte Kollektivgüter, die Gruppenkollektivgüter, nicht vom Staat, sondern von Parafiski (also insbesondere karitativen Organisationen) bereitgestellt werden sollten, da nur so institutionelle Kongruenz bzw. fiskalische Äquivalenz gewährleistet werden könne - deren Fehlen für einen Großteil der heute zu beobachtenden sozialpolitischen Probleme verantwortlich sei. 
Der geldpolitische Teil unserer Festschrift steht ganz im Zeichen des Euro bzw. der europäischen Geldpolitik. Er wird eröffnet mit Kapital 15 („,Politische Glaubwürdigkeit und der Euro: Eine verfassungsökonomische Perspektive") von Klaus W. Zimmermann und Tobias Just, die der Frage nachgehen, warum eine so wichtige Entscheidung, wie sie die Einführung des Euro war, dezidiert gegen den Willen der großen Mehrheit der Bevölkerung getroffen wurde. Sie stellen fest, daß nur eine Minderheit vom Euro (ökonomisch) profitiert, und erläutern anhand eines verfassungsökonomischen Modells, warum sich die Gewinner-Minderheit nicht auf ein Euroreferendum eingelassen hat: entweder, weil sie die Verlierer-Mehrheit nicht entschädigen wollte, oder, weil sie dies nicht konnte (d.h. weil der Gesamtnutzen der Euroeinführung unter deren Gesamtkosten liegt). In jedem Fall sei die ,undemokratische“ Euroeinführung in Deutschland der Akzeptanz nicht nur der neuen Währung, sondern auch des politischen Systems nicht gerade förderlich gewesen.

Wesentlich für die künftige Akzeptanz des Euro wird dessen (Binnen-) Stabilität sein. Egon Görgens warnt in Kapitel 16 (,Mögliche Konflikte zwischen Geld- und Finanzpolitik in der Europäischen Währungs- und Wirtschaftsunion") vor der Gefährdung der Preisniveaustabilität durch übermäßige Haushaltsdefizite. Der Autor sieht im Stabilitäts- und Wachstumspakt, der der Staatsverschuldung der Euroländer Grenzen setzen soll, zwar eine wichtige Maßnahme zur Vorbeugung gegen solche Konflikte zwischen Geld- und Finanzpolitik. Er bezweifelt aber, ob dieser Pakt auf Dauer und auch unter ungünstigen makroökonomischen Bedingungen die in ihn gesetzten Erwartungen erfüllen kann.

Eingehender mit dem Stabilitäts- und Wachstumspakt befassen sich Heinz-Dieter Smeets und Bernard Vogl in Kapitel 17 (,Der Stabilitäts- und Wachstumspakt - eine kritische Würdigung"). Sie halten die Disziplinierung der nationalen Haushaltspolitiken in der Europäischen Währungsunion für grundsätzlich notwendig, kritisieren aber das im Stabilitäts- und Wachstumspakt geregelte Verfahren als zu langwierig und zu sehr politisiert. Nicht stichhaltig sei dagegen der Einwand, der Pakt würde zu einer übermäßigen Einengung des finanzpolitischen Spielraums führen; vielmehr würde - bei erfolgreicher Konsolidierung der nationalen Haushalte - der (sinnvolle) Einsatz automatischer Fiskalstabilisatoren kaum eingeschränkt werden.

Wenn man einmal davon ausgeht, daß die Europäische Zentralbank ihre Geldpolitik nicht nur de jure, sondern auch de facto autonom, d.h. nicht unter dem Druck der Haushaltspolitik betreiben kann, stellt sich die Frage, welche geldpolitische Strategie gewählt werden sollte. Aufgrund ihres Erfolgs in Sachen Preisniveaustabilität ziehen Bernhard Herz und Claus Grei- 
ber in Kapitel 18 („Taylor-Regeln: Erfahrungen der Deutschen Bundesbank, Implikationen für die Europäische Zentralbank") die Deutsche Bundesbank als Vorbild für die Europäische Zentralbank heran. Sie analysieren die Geldpolitik der Deutschen Bundesbank mit Hilfe von Taylor-Regeln und leiten aus deren Erfahrungen Empfehlungen für die Europäische Zentralbank ab: Sie sollte ihre Geldpolitik vor allem an den Abweichungen der tatsächlichen von der angestrebten Inflationsrate ausrichten und dabei auch den durchaus bestehenden Spielraum zur Konjunkturstabilisierung nutzen.

Mit einer beruhigenden Botschaft beschließt Arno Wilfert in Kapitel 19 („Elektronisches Geld - enttäuschte Erwartungen und übertriebene Befürchtungen") den geldpolitischen Teil dieses Bandes: Welche Gefahren dem Euro auch immer drohen mögen - die durch elektronisches Geld gehört nicht dazu. Bis vor kurzem wurden die Risiken des elektronischen Geldes für die Geldpolitik - insbesondere der Verlust der Kontrolle über die Geldmenge - intensiv diskutiert. Doch diese Befürchtungen hätten sich als übertrieben erwiesen. Aufgrund der bislang sehr geringen Verbreitung des elektronischen Geldes und der Ausdehnung der Autorität von Zentralbanken und Bankenaufsichten auf die Emittenten dieser Geldform, müsse sich die Europäische Zentralbank zumindest in dieser Hinsicht auf absehbare Zeit keine Sorgen machen.

Allen Autoren danken wir ganz herzlich für ihre Mitarbeit und für ihre Geduld mit unseren Änderungswünschen und Ergänzungvorschlägen. Eine wertvolle Hilfe war uns Sylvia Mette vom Lehrstuhl für Finanzwissenschaft an der Technischen Universität Ilmenau, die das Layout des Gesamtmanuskripts in gewohnt professioneller Weise gestaltet hat. Bedanken möchten wir uns außerdem bei Dr. Martina Bihn und Dr. Werner A. Müller vom Springer- bzw. Physica-Verlag für die (wie immer) angenehme Zusammenarbeit. Schließlich darf die finanzielle Unterstützung dieses Projekts durch den Universitätsverein Bayreuth $\mathrm{eV}$ und den Verein zur Förderung der Forschungsstelle für Bankrecht und Bankpolitik der Universität Bayreuth $\mathrm{eV}$ keinesfalls unerwähnt bleiben.

$\mathrm{Zu}$ guter Letzt möchten wir Ihnen, lieber Dieter Fricke, auch im Namen aller Autoren, recht herzlich zum 65. Geburtstag gratulieren und Ihnen viel Freude bei der Lektüre Ihrer Festschrift sowie alles Gute für Ihren weiteren Lebensweg wünschen. 


\section{Inhaltsverzeichnis}

DIETER FRICKE ZUM 65. GEBURTSTAG - V

INHALTSVERZEICHNIS - -

\section{Neue Herausforderungen für die Finanzpolitik}

1. DER DRITTE SEKTOR - EINE CHANCE FÜR DEN STAAT?--1 Guy Kirsch

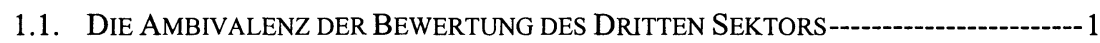

1.2. DER DRITTE SEKTOR ALS NICHTSTAATLICHE UND NICHTMERKANTILE

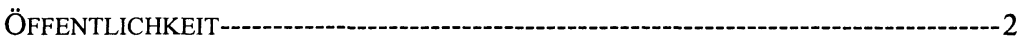

1.3. ZUM VERHÄLTNIS VON MARKT, STAAT UND DRITTEM SEKTOR ---:----:--------5

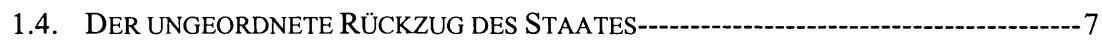

1.5. Die ENTSTAATlichung DER GeSEllschaft - UND WARUM SIE (FAST) NICHT ZUSTANDEKOMMT --.-- 10

1.6. INSTITUTIONEN: ORTE UND ERGEBNISSE VON VERTEILUNGSKONFLIKTEN ------ 11

1.7. AUSZAHLUNGSMATRIZEN-- 13

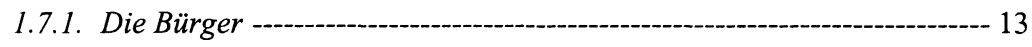

1.7.2. Die Politiker - 15

1.7.3. Die Verwaltung -- 17

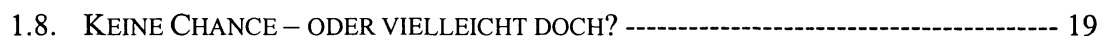

2. DIE ENTWICKLUNG DER STEUERSTRUKTUREN IM

INTERNATIONALEN VERGLEICH- 21

Dieter Ewringmann und Klaus Mackscheidt

2.1. EINIGE VORBEMERKUNGEN ZUR STEUERDISKUSSION -- 21

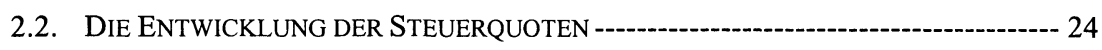

2.3. DIE ENTWICKLUNG DER STEUERSTRUKTUREN - 28

2.4. VERSCHIEBUNGEN IM BEREICH DER INDIREKTEN STEUERN

- DIE ROLLE DER ÖKOSTEUERN -- 35

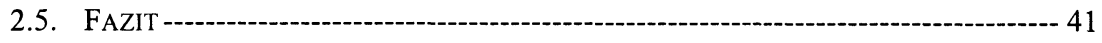

2.6. LITERATUR - 43 
3. DIE UNTERNEHMENSBESTEUERUNG - REFORMSTAND UND

REFORMBEDARF

Jochen Sigloch

3.1. EINFÜHRUNG

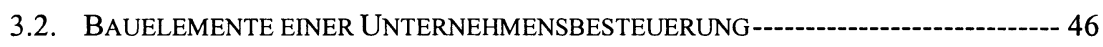

3.2.1. Unternehmensabgrenzung und -rechtsform---- 47

3.2.2. Technisches Unternehmenssteuersystem - 47

3.2.3. Einsteuer-versus Mehrsteuersystem -

3.2.4. Erfolgsmaßgrößen und Ermittlungsmodelle --_-_- 49

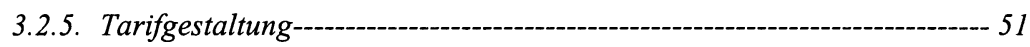

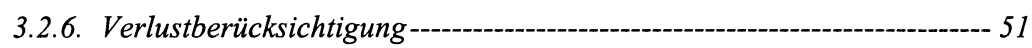

3.2.7. Veräußerungsgewinnbesteuerung ------- 52

3.3. ANALYSE DER BISHERIGEN REFORM DER UNTERNEHMENSBESTEUERUNG------- 53

3.3.1. Unternehmensabgrenzung und -rechtsform---o- 53

3.3.2. Technisches Unternehmenssteuersystem ---------------------------- 54

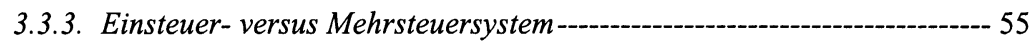

3.3.4. Erfolgsmaßgrößen und Ermittlungsmodelle ----------------------- 57

3.3.5. Tarifgestaltung---- 58

3.3.6. Sonderregelungen zur Verlustberücksichtigung und

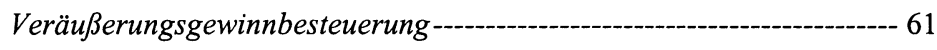

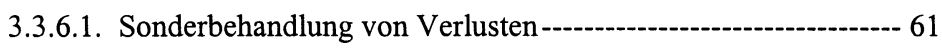

3.3.6.2. Sonderbehandlung von Veräußerungsgewinnen -------------- 61

3.3.7. Ergebnis --- 62

3.4. ANSATZPUNKTE FÜR EINE SYSTEMATISCHE REFORM DER

UNTERNEHMENSBESTEUERUNG -- 63

3.4.1. Unternehmensabgrenzung und -rechtsform - 63

3.4.2. Technisches Unternehmenssteuersystem ---------------------------- 65

3.4.3. Einsteuer-versus Mehrsteuersystem-- 65

3.4.4. Erfolgsmaßgrößen und Ermittlungsmodelle ---------------------------- 66

3.4.5. Tarifgestaltung-- 68

3.4.6. Sonderregelungen zur Verlustberücksichtigung und

Veräußerungsgewinnbesteuerung --- 69

3.4.6.1. Sonderbehandlung von Verlusten -

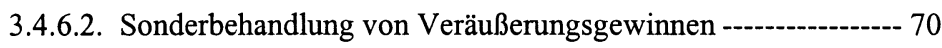

3.4.7. Ergebnis -- 71

3.5. ABSCHLIESSENDE BEMERKUNGEN- 72

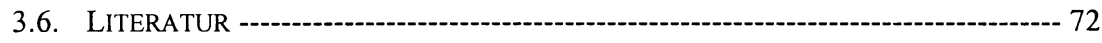


4. DER BEIHILFENBERICHT DER EUROPÄISCHEN UNION:

DARSTELLUNG UND BEWERTUNG

Dietrich Dickertmann und Annemarie Leiendecker

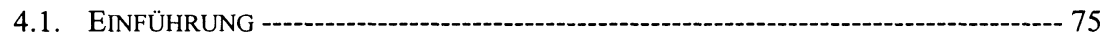

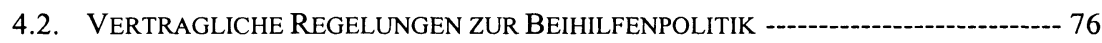

4.3. CHRONOLOGIE DER BEIHILFENBERICHTERSTATTUNG --- 80

4.3.1. Beihilfen im Bericht über die Wettbewerbspolitik-- 81

4.3.2. Vorlage eines eigenständigen Beihilfenberichts-- 83

4.4. ANLAGE UND AUfBAU DES ACHTEN BEIHILFENBERICHTS---:-

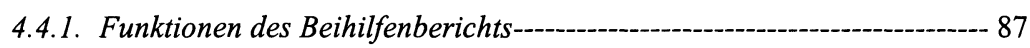

4.4.2. Zweckbestimmungen der Beihilfen --

4.4.3. Nationale Beihilfen - Ergebnisse --_- 92

4.4.4. Aussagen über die Gemeinschaftshilfen - im Anhang ---_-_-- 95

4.5. METHODIK DER BERICHTERSTATTUNG-- 99

4.5.1. Erfassungsbereich

4.5.2. Datenquellen - 100

4.5.3. Aufbereitung und Verarbeitung der Daten---- 102

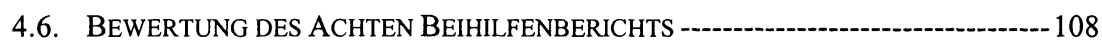

4.6.1. Beurteilungskriterien -- 108

4.6.1.1. Informationsfunktion --- 109

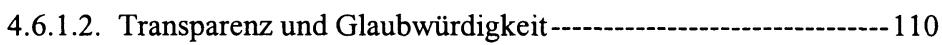

4.6.2. Formale Beurteilung --- 112

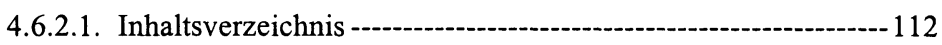

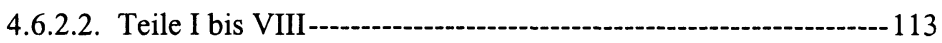

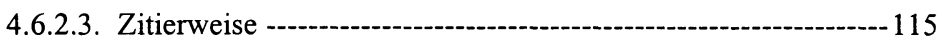

4.6.2.4. Genauigkeit --

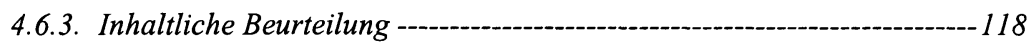

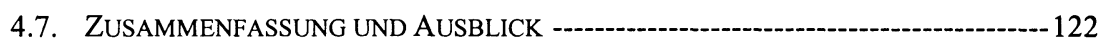

4.8. LITERATUR - 126

5. ÖFFENTLICHE UNTERNEHMEN IN DER MARKTWIRTSCHAFT:

DAS BEISPIEL EISENBAHN

Helmut Gröner und Andreas Knorr

5.1. EINLEITUNG

5.2. ORDNUNGSTHEORETISCHE GRUNDLAGEN --131

5.2.1. Grundprinzipien marktwirtschaftlicher Ordnungen ---------131

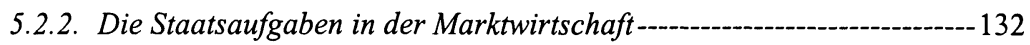

5.2.3. Zur Problematik öffentlicher Unternehmen als Mittel der

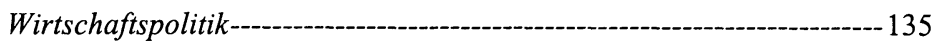

5.3. EINE KURZE HISTORIE DER EISENBAHNPOLITIK IN DEUTSCHLAND ---.---.-----137 
5.3.1. Von den Anfängen bis zur Gründung der Reichsbahn----------------137

5.3.2. Die Zwischenkriegszeit --- 140

5.3.3. 1945 bis zur Bahnstrukturreform 1994 ---o- 142

5.3.4. Von der Bahnstrukturreform 1994 bis heute-- 149

5.4. KRITISCHE WÜRDIGUNG --152

5.4.1. Allgemeine Schwächen des deutschen Staatsbahnmodells bis zur

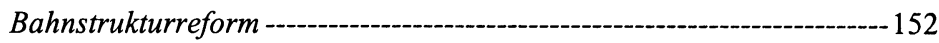

5.4.2. Warum scheiterte die Eisenbahnschutzpolitik? --- 153

5.4.3. Ordnungspolitische Mängel der Bahnstrukturreform ----------154

5.4.4. „Unfaire“ Wettbewerbsvorteile anderer Verkehrsträger?------------155

5.5. SCHLUSSBETRACHTUNG UND AUSBLICK --- 157

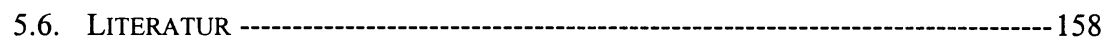

6. EIN HEBESATZRECHT FÜR LÄNDER UND GEMEINDEN? Horst Zimmermann

6.1. DIE PROBLEMLAGE -

6.2. BEWERTUNGSMASSSTÄBE: VOM FiSKALFÖDERALISMUS ZUM

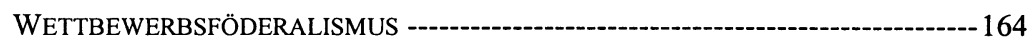

6.2.1. Eine statische Betrachtung: Fiskalföderalismus --- 164

6.2.2. Die dynamische Ergänzung: Wettbewerbsföderalismus--------------166

6.3. HANDLUNGSERFORDERNISSE IN DER BUNDESREPUBLIK DEUTSCHLAND:

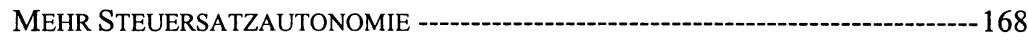

6.3.1. Der Hintergrund: Starke Betonung der regionalen Einheitlichkeit----- 168

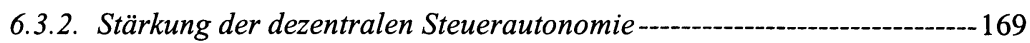

6.4. DIE WAHL DES EINNAHMESYSTEMS -

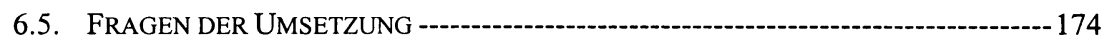

6.6. LITERATUR - 177

7. EIN WEG AUS DER KRISE DES DEUTSCHEN

LÄNDERFINANZAUSGLEICHS---181

Fritz Söllner

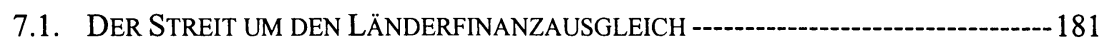

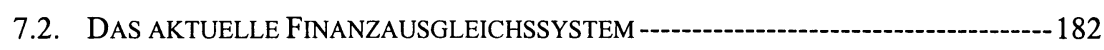

7.2.1. Überblick --

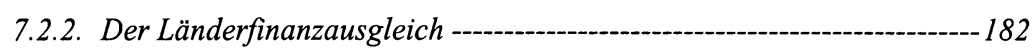

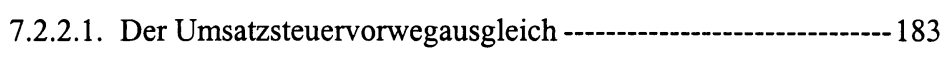

7.2.2.2. Der Länderfinanzausgleich im engeren Sinne----------------- 184

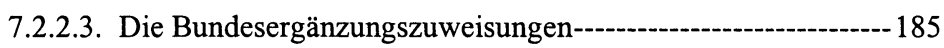

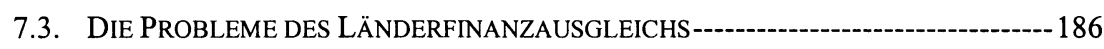

7.3.1. Der Umsatzsteuervorwegausgleich -------186 


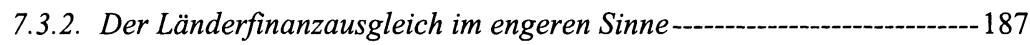

7.3.3. Die Bundesergänzungszuweisungen ---_- 189

7.3.4. Die Gesamtwirkung - 191

7.4. DAS URTEIL DES BUNDESVERFASSUNGSGERICHTS

VOM 11. NOVEMBER 1999 - 196

7.5. KONSEQUENZEN FÜR DEN LÄNDERFINANZAUSGLEICH:

EIN REFORMVORSCHLAG - - 198

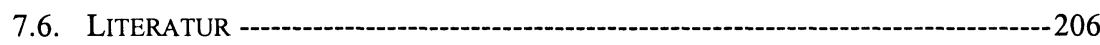

8. DER EINFLUSS DES SOZIALSTAATSPRINZIPS AUF DIE

ENTWICKLUNG DES DEUTSCHEN LÄNDERFINANZAUSGLEICHS----208

Peter Thuy

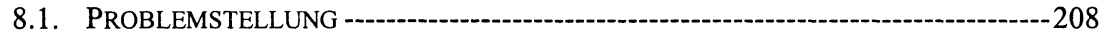

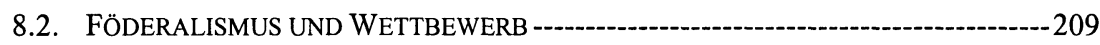

8.2.1. Grundzüge der Wettbewerbsgesellschaft-- 209

8.2.2. Föderalismus und Wettbewerb in der Bundesrepublik Deutschland --- 210

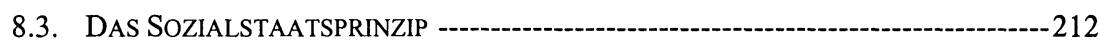

8.3.1. Grundzüge-- 212

8.3.2. Interpretationsmöglichkeiten --- 212

8.3.3. Sozialstaat und Wettbewerb-- 215

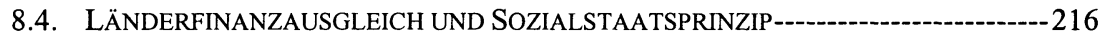

8.4.1. Historische Enwicklung ---216

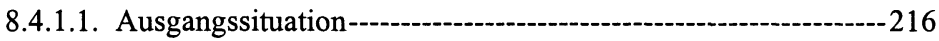

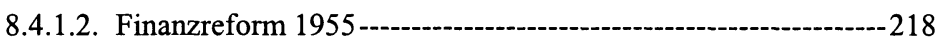

8.4.1.3. Finanzreform 1969--on

8.4.2. Entwicklung der Zahlungsströme --

8.4.3. Wirkungen des Finanzausgleichs

8.5. DAS SozIALSTAATSPRINZIP ALS URSACHE FÜR DIE KRISE DES

LÄNDERFINANZAUSGLEICHS ?---:-_- 222

8.6. LITERATUR -

\section{Der Sozialstaat in der Krise}

9. SOZIALSTAATSPRINZIP UND WIRTSCHAFTSORDNUNG Peter Oberender und Stephan Ruckdäschel

9.1. EINLEITUNG - -

9.2. STAATSZIELE IM GRUNDGESETZ: RECHTS- UND SOZIALSTAATSPRINZIP-------- 228

9.2.1. Der Rechtsstaat im Grundgesetz --- 228

9.2.2. Der Sozialstaat im Grundgesetz --- 229

9.2.3. Die Gesamtentscheidung für den Sozialen Rechtsstaat-------------_---229 
9.3. Die SOZIALE MARKTWIRTSCHAFT ALS MÖGLICHE WiRTSCHAFTSORDNUNG --- 230

9.3.1. Grundgesetz und Wirtschaftsordnung -- 230

9.3.2. Wettbewerb in der Sozialen Marktwirtschaft -------------------230

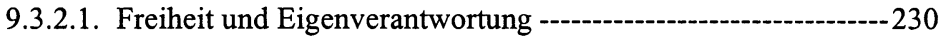

9.3.2.2. Menschenbild --

9.3.3. Die Soziale Marktwirtschaft --_--_---233

9.4. Die RolLE DES STAATES -

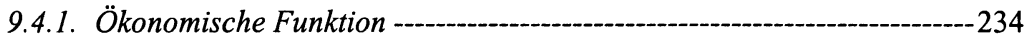

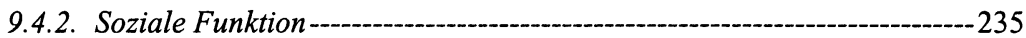

9.4.3. Nutzen und Kosten sozialstaatlicher Tätigkeit------------------------236

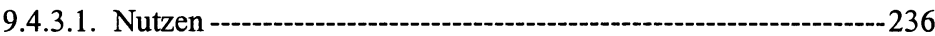

9.4.3.2. Kosten --

9.4.3.3. Zwischenergebnis --- 237

9.4.3.4. Formen sozialstaatlicher Tätigkeit --

9.5. HANDLUNGSRICHTLINIEN FÜR DEN SOZIALSTAAT ---

9.5.1. Vorrang der Eigenverantwortlichkeit--_- 240

9.5.2. Ordnungskonformität --- 241

9.5.3. Zielkonformität --

9.5.4. Konstanz der Sozialpolitik -

9.6. SCHLUSSBEMERKUNG --- 242

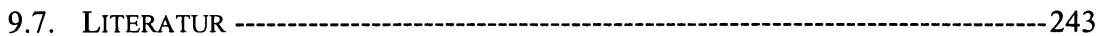

10. ZUR NOTWENDIGKEIT EINER HARMONISIERUNG DER STAATLICHEN KRANKENVERSICHERUNGSSYSTEME IN EUROPA---246 Frank Daumann

10.1. PROBLEMSTELLUNG UND VORGEHENSWEISE---

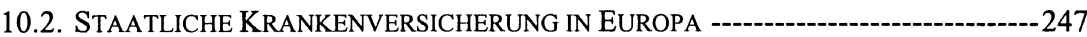

10.3. TRADITIONELLE ANSÄTZE ZUR BEURTEILUNG DER NOTWENDIGKEIT EINER

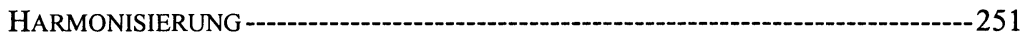

10.3.1.Harmonisierung im integrationstheoretischen Kontext ---

10.3.2. Harmonisierung im Kontext des Bereitstellungkostenansatzes--_-_----253

10.3.3. Harmonisierung im Kontext des

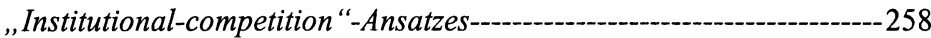

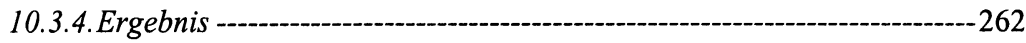

10.4. STAATLICHE KRANKENVERSICHERUNGSSYSTEME BEI FREIEM GÜTER- UND

FAKTORVERKEHR

10.4.1.Kriterien einer Beurteilung der Notwendigkeit

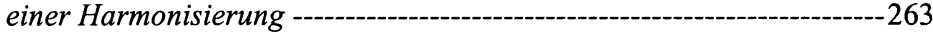

10.4.1.1.Operationalisierung des Kriteriums Legitimation---------------263

10.4.1.2.Operationalisierung des Kriteriums Finanzierbarkeit ----------264 
10.4.2.Zur Legitimation staatlicher Krankenversicherungssysteme ------------265

10.4.3. Zur Finanzierbarkeit sozialer Krankenversicherungssysteme-----------265

10.4.3.1.Szenario I: Unbeschränkte Wechselmöglichkeit und regional unbeschränkte Leistungsinanspruchnahme-------------266

10.4.3.2.Szenario II: Unbeschränkte Wechselmöglichkeit und regional beschränkte Leistungsinanspruchnahme-----------267

10.4.3.3.Szenarien III und IV: Beschränkte Wechselmöglichkeit-------268

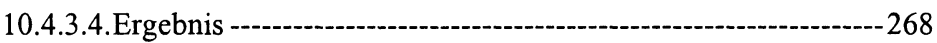

10.5. ANSÄTZE ZUR AUFLÖSUNG DER FUNKTIONELLEN UNDIFFERENZIERTHEIT

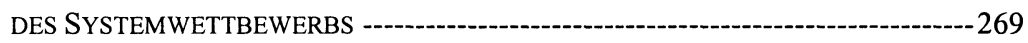

10.5.1. Heimathafenprinzip -- 270

10.5.2. Wahlfreiheit bei Beginn der Beschäftigung -------------------270

10.5.3. Wahlfreiheit mit frei vereinbarten Kompensationszahlungen -----------271

10.6. FAZIT -- 273

10.7. LITERATUR -

11. DIE RENTENREFORM IN DEUTSCHLAND - EINE KRITISCHE

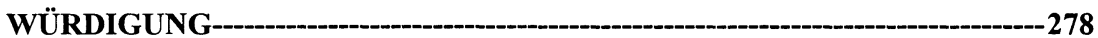

Bert Rürup

11.1. GRÜNDE FÜR DIE NOTWENDIGKEIT EINES UMBAUS DER RENTEN-

VERSICHERUNG-- 278

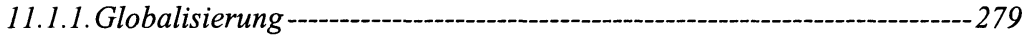

11.1.2.Informatisierung-- 280

11.1.3.Alterung - - 282

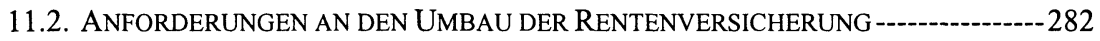

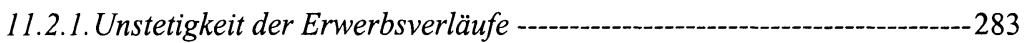

11.2.2.Alterung --

11.2.2.1. Verlängerung der Lebensarbeitszeit --- 284

11.2.2.2. Verschiebung der Finanzierungsrelationen ---

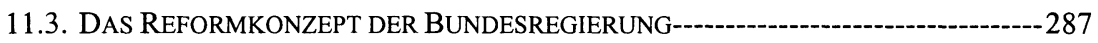

11.3.1. Beschreibung des Reformkonzepts -- 287

11.3.2. Die private Altersvorsorge - freiwillig oder verpflichtend? ---_------292

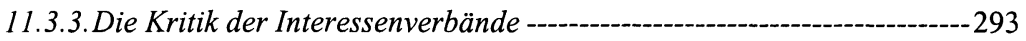

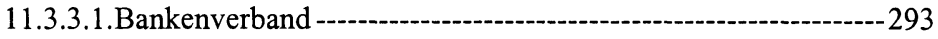

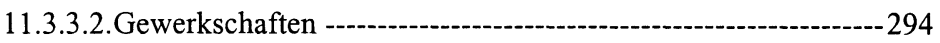

11.4. FAZIT -

11.5. LITERATUR - 
12. WEGE ZU MEHR BESCHÄFTIGUNG IN DEUTSCHLAND AUSLÄNDISCHE ERFAHRUNGEN ALS VORBILD? 297

Klaus Schuberth

12.1. DiE ARBEITSMARKTENTWICKLUNG IN DER BUNDESREPUBLIK

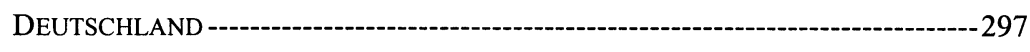

12.2. URSACHEN DER ARBEITSLOSIGKEIT - 300

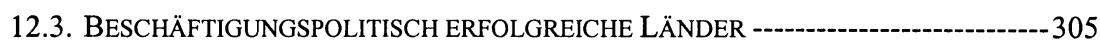

12.3.1.Die Vereinigten Staaten -

12.3.2.Die Niederlande -

12.3.3. Großbritannien -- 310

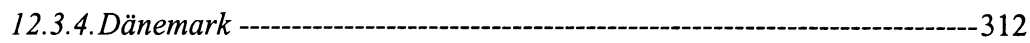

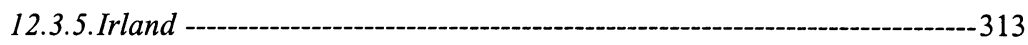

12.4. SCHLUSSFOLGERUNGEN AUS DEN ERFAHRUNGEN ANDERER LÄNDER-----------315

12.5. MEHR BESCHÄFTIGUNG UND WENIGER ARBEITSLOSIGKEIT

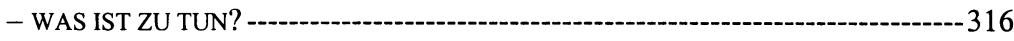

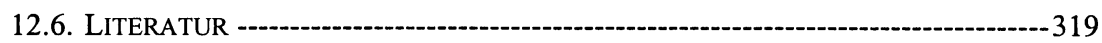

13. DIE STAATLICHE ZWANGSSCHLICHTUNG ALS AUSWEG AUS

TARIFKONFLIKTEN?

Thomas Pfahler

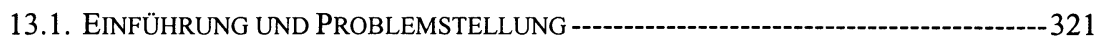

13.2. THEORETISCHE GRUNDLAGEN --- 322

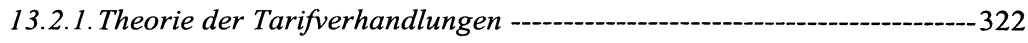

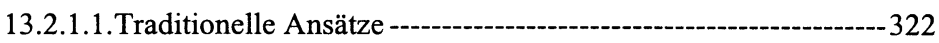

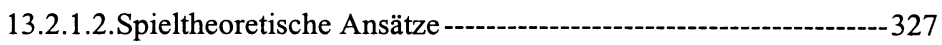

13.2.1.3.Zusammenfassung --- 328

13.2.2. Theorie der Schlichtung --- 329

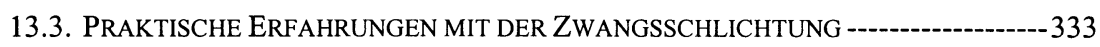

13.3.1.Erklärungsrelevante Daten -- 333

13.3.2. Anfänge des Schlichtungswesens in Deutschland ---------------335

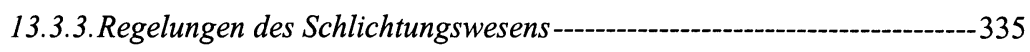

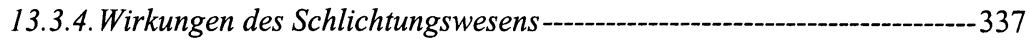

13.4. FAZIT ---

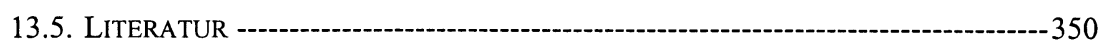

14. KARITATIVE ORGANISATIONEN UND DIE KRISE DES

SOZIALSTAATS

Klaus Tiepelmann und Gregor van der Beek

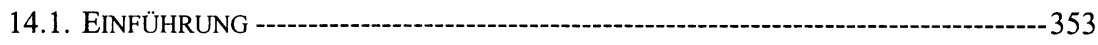

14.2. KARITATIVE SELBST- UND FREMDHILFEGRUPPEN IN DEUTSCHLAND ----------354 
14.3. KARITATIVE GRUPPEN IN DER „NONPROFIT“-THEORIE 356

14.4. PARAFISKALISCHE INSTITUTIONEN UND DIE KÖLNER SCHULE DER FINANZWISSENSCHAFT 360

14.5. DiE KRISE DES SOZIALSTAATS IM LICHTE DES FINANZTHEORETISCHEN ZUGANGS ZU PARAFISKI 361

14.6. SOZIALPOLITIK IN DEN NICHT FINANZÖKONOMISCHEN ZUGÄNGEN ZU PARAFISKI---

14.7. FAZIT --

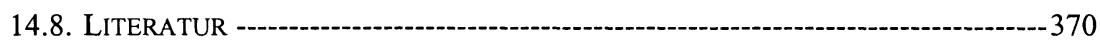

\section{Der Euro und die europäische Geldpolitik}

15. POLITISCHE GLAUBWÜRDIGKEIT UND DER EURO: EINE VERFASSUNGSÖKONOMISCHE PERSPEKTIVE $-373$

Klaus W. Zimmermann und Tobias Just

15.1. EINLEITUNG 373

15.2. DER POLITÖKONOMISCHE HINTERGRUND DES EURO $-375$

15.2.1. Kosten und Nutzen einer Währungsunion $-375$

15.2.2. Befürworter der Währungsunion in Deutschland --

15.3. DAS VERFASSUNGSÖKONOMISCHE KALKÜL --

15.3.1.Die verfassungsrechtliche Möglichkeit eines Referendums ---_--------380

15.3.2. Das Informationsproblem bei Referenda-- 382

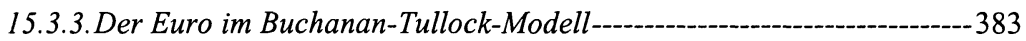

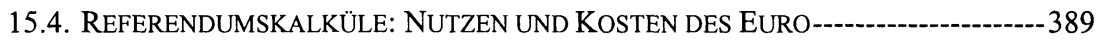

15.5. SCHLUSSBEMERKUNGEN --

15.6. LITERATUR --.--_-

16. MÖGLICHE KONFLIKTE ZWISCHEN GELD- UND FINANZPOLITIK IN DER EUROPÄISCHEN WÄHRUNGS- UND WIRTSCHAFTSUNION----398 Egon Görgens

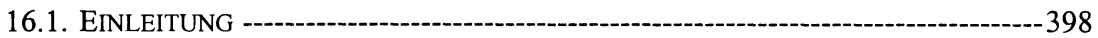

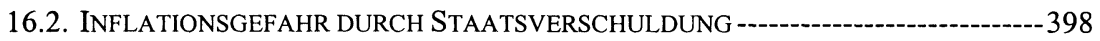

16.3. KONFLIKTPOTENTIALE UNTERSCHIEDLICHER

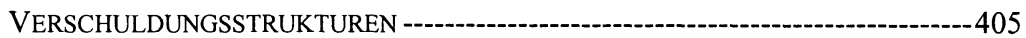

16.4. UNKOORDINIERTE GELD- UND FINANZPOLITIKEN --

16.4.1.Der Stabilitäts- und Wachstumspakt--

16.4.2. Das finanzpolitische Doppelkriterium -

16.5. ABSCHLIESSENDE BEMERKUNGEN--

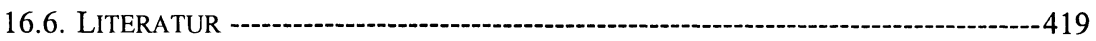


17. DER STABILITÄTS- UND WACHSTUMSPAKT - EINE KRITISCHE WÜRDIGUNG

Heinz-Dieter Smeets und Bernard Vogl

17.1. EINLEITUNG

17.2. VERÄNDERTE RAHMENBEDINGUNGEN FÜR DIE FISKALPOLITIK IN DER EWU

17.3. DIE FINANZPOLITISCHEN VORGABEN DES STABILITÄTS- UND

WACHSTUMSPAKTS-

17.4. EINSCHRÄNKUNG DER BUDGETFLEXIBILITÄT DURCH DEN STABILITÄTSUND WACHSTUMSPAKT?

17.5. KRITISCHE WÜRDIGUNG

17.6. LITERATUR

18. TAYLOR-REGELN: ERFAHRUNGEN DER DEUTSCHEN

BUNDESBANK, IMPLIKATIONEN FÜR DIE

EUROPÄISCHE ZENTRALBANK

Bernhard Herz und Claus Greiber

18.1. PROBLEMSTELLUNG 453

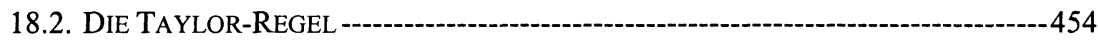

18.3. SCHÄTZUNGEN DER TAYLOR-REGEL -

18.3.1.Das Modell --

18.3.2.Ergebnisse---

18.3.3. Zusammenfassung der empirischen Ergebnisse -_-_-_-_-_-_-_

18.4. SCHLUSSFOLGERUNGEN--

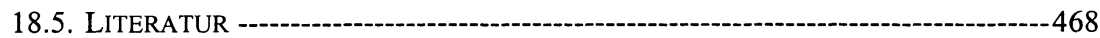

19. ELEKTRONISCHES GELD - ENTTÄUSCHTE ERWARTUNGEN

UND ÜBERTRIEBENE BEFÜRCHTUNGEN -

Arno Wilfert

19.1. EINLEITUNG

470

19.2. FORMEN ELEKTRONISCHEN GELDES -

19.2.1.Elektronische Geldbörsen - 472

19.2.2.Netzgeld-- 478

19.3. DIE VERBREITUNG ELEKTRONISCHEN GELDES --

19.4. GELDPOLITISCHE RISIKEN VON ELEKTRONISCHEM GELD - -

19.5. AUSBLICK: DIE BARGELDLOSE GESELLSCHAFT IN WEITER FERNE? ------.-------486

19.6. LITERATUR - 